ORIGINAL ARTICLE

\title{
Effects of prolonged strenuous exercise (marathon running) on biochemical and haematological markers used in the investigation of patients in the emergency department
}

\author{
J E Smith, G Garbutt, P Lopes, D Tunstall Pedoe
}

Br J Sports Med 2004;38:292-294. doi: 10.1136/bjsm.2002.002873

See end of article for authors' affiliations

Correspondence to: Jason E Smith, Academic Department of Sports Medicine, Royal London Hospital (Mile End), Bancroft Road, London El, UK; jason.smith75@ virgin.net

Accepted 17 March 2003

\begin{abstract}
Objectives: To investigate the effects of strenuous exercise on commonly used biochemical and haematological variables in subjects running the 2002 London marathon.

Methods: 34 healthy volunteers ( 7 female, 27 male) were recruited for the study. Blood was taken before the start (at registration) and immediately after completion of the marathon. Samples were analysed for urea and electrolytes, liver function tests, creatine kinase (CK), CK-MB isoenzyme, myoglobin, troponin I, full blood count, a clotting screen, and D-dimers. The results before and after exercise were compared. Pearson's correlation coefficients were calculated for all variables.

Results: Significant increases were found in CK, CK-MB, aspartate aminotransferase (AST), lactate dehydrogenase (LDH), and myoglobin following the marathon. However, there was no significant change in the level of troponin I. There was also evidence of activation of the coagulation and fibrinolytic cascades following the marathon, with a reduction in activated partial thromboplastin time, a reduction in fibrinogen, and an increase in D-dimers.

Conclusions: The results confirm previous individual studies on marathon running and the biochemical and haematological tests routinely carried out in hospital. These are affected by prolonged exercise, and "abnormal" results in these tests may be normal after prolonged exercise and therefore not diagnostic of a disease process. The results of investigations in patients who have been exercising should be interpreted with caution.
\end{abstract}

iochemical and haematological measurements form the basis of the initial investigation of several clinical problems in patients who may have undertaken recent exercise, including cardiac chest pain, exertional heat stroke, and thromboembolic disease.

The traditional markers of cardiac damage are the enzymes creatine kinase (CK), aspartate aminotransferase (AST), and lactate dehydrogenase (LDH), but recently, more specific markers of myocardial damage have been identified. These include the CK-MB isoenzyme, myoglobin, and cardiac troponins. Many emergency departments and chest pain assessment units use measurement of these variables to rule out or rule in myocardial infarction. ${ }^{1}$

In patients with suspected thromboembolic disease in the form of pulmonary embolism or deep vein thrombosis, clinical risk stratification occurs in conjunction with investigation. Many protocols for the investigation and treatment of patients with possible deep vein thrombosis or pulmonary embolism include measurement of D-dimers as part of the investigative process. ${ }^{23}$

It has been shown that exercise may influence the results of these investigations in asymptomatic healthy subjects, particularly if the exercise is prolonged or strenuous. ${ }^{4}$ Many of the studies in this area have used laboratory based subjects. In the present study, we aimed to quantify these effects in a group of runners completing the Flora London marathon on 14 April 2002.

\section{METHODS}

Before the study, ethical approval was sought and obtained from the East London and the City Health Authority (ELCHA) research ethics committee. All volunteers provided informed written consent before participation. Thirty eight healthy amateur runners aged between 18 and 65 years were recruited, and pre-race (control) blood samples were taken at registration, one to three days before the race, following completion of a short questionnaire on training status and medical history. Potential participants with a history of muscle disorder, clotting abnormality, or cardiac disease, and those taking drugs that may have altered the measured variables were excluded. No limitation was placed on the amount of fluid subjects could consume during the race.

Thirty four of the runners finished the race within five hours $(>8.4 \mathrm{~km} / \mathrm{h})$ and attended the finish area for post-race blood sampling within an hour of finishing. These formed our study group. Blood samples were taken from an antecubital vein using the Monovette ${ }^{\circledR}$ vacuum system and a sterile technique. Approximately $20 \mathrm{ml}$ of blood was collected in four tubes: a clotted sample tube for biochemistry, an EDTA tube for haematology, a lithium heparin tube for cardiac panel testing, and a citrate tube for clotting analysis. To eliminate errors from postural changes in plasma volume, ${ }^{6}$ all subjects were sampled in the upright sitting position, both before and after the race. Sample analysis was done on site using a near-patient testing analyser for CK-MB, myoglobin, and troponin I (Triage ${ }^{\circledR}$ Cardiac Panel, Biosite Diagnostics Inc, San Diego, California, USA). Samples for the remaining investigations were taken to the pathology laboratory at the Homerton Hospital, London, where all samples were analysed within four hours of venepuncture. Pre- and post-race samples were analysed for biochemistry (urea and electrolytes, liver function tests, CK, CK-MB, myoglobin, and troponin I) and haematology (full blood count, clotting screen, fibrinogen, and D-dimers). Changes in plasma volume $(\triangle \mathrm{PV})$ were calculated using the method described by Van Beaumont et al. ${ }^{7}$ The pre- and post-race results were compared using a paired $t$ test with SPSS 11.0 for 


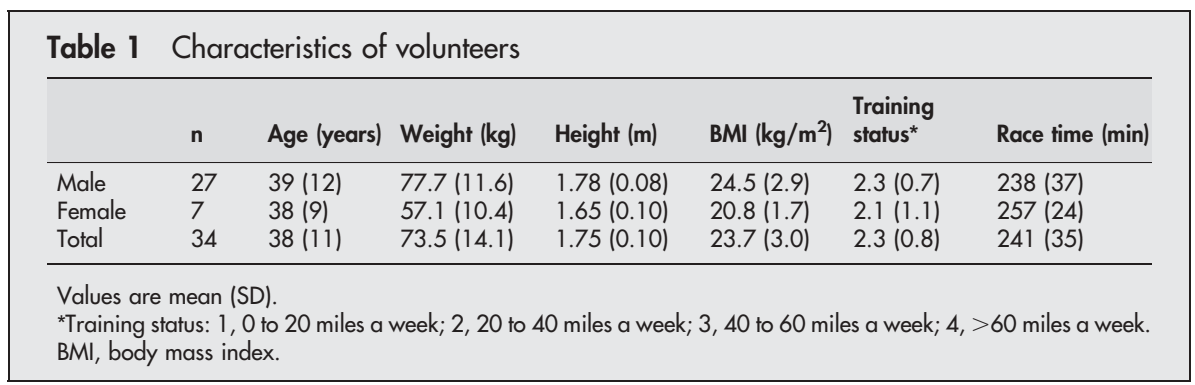

Windows software. Numerical data are presented as mean (SD), and $\mathrm{p}$ values of $<0.05$ are considered statistically significant.

\section{RESULTS}

There were 27 male and seven female participants, and their characteristics are summarised in table 1. The results (with units of measurement) are given in table 2. The mean $\Delta \mathrm{PV}$ was $+2.5(5.7) \%$, suggesting minor haemodilution in most runners. Near-patient testing revealed a significant $(p<0.001)$ rise in CK-MB following exercise (3.6 (2.2) U/l pre- versus 13.5 (9.6) post-exercise), a rise in myoglobin (all but one post-exercise results were $>500 \mathrm{ng} / \mathrm{ml}$ ), but no significant increase in troponin I. Indeed, the troponin I results all remained $<0.3 \mu \mathrm{g} / \mathrm{l}$, well below the upper limit of normal range. Although the rise in CK-MB was significant in absolute terms, the percentage of total CK post-exercise never exceeded $5 \%$ (mean $2.0(0.9) \%$, range $0.53 \%$ to $4.41 \%$ ). Significant correlations were found between training status $(p<0.05)$, faster race time $(p<0.01)$, and both CK and CKMB pre-exercise. The percentage change in CK following exercise was positively correlated with lower training status and slower race time $(\mathrm{p}<0.05)$. The white blood cell count rose significantly $(\mathrm{p}<0.001)$ following exercise $(6.4$ (1.4) $\times 10^{9} / 1$ pre $v 17.4$ (3.6) post), predominantly because of a neutrophilia. Clotting studies showed evidence of activation of both the coagulation and fibrinolytic cascades, with reduced APTT, reduced levels of fibrinogen (even taking into account changes in plasma volume there was still a $-2.94 \%$ change in fibrinogen concentration) and increased D-dimers (234.0 (130.6) ng/ml pre versus 553.7 (378.6) post, $\mathrm{p}<0.001)$.

\section{DISCUSSION}

This study confirms that many of the biochemical and haematological tests routinely carried out in hospital are affected by exercise in the form of marathon running, and abnormalities in these tests may be a consequence of exercise rather than of a disease process.

Studies in the late 1970s and early 1980s showed that CK and CK-MB are raised following marathon running. ${ }^{8}$ Indeed, the traditional cardiac markers CK, AST, and LDH are all seen to rise beyond the normal range following exercise, as are the more specific cardiac markers such as CK$\mathrm{MB}$ and myoglobin. However, when calculated as a percentage of total CK, the CK-MB did not rise above $4.4 \%$ following exercise in this study, confirming that the rise in this isoenzyme originated from skeletal muscle rather than cardiac muscle.

More recently work has been carried out to investigate the effects of exercise on the newer cardiac markers such as the cardiac troponins. In contrast to some studies, ${ }^{10}{ }^{11}$ troponin I was not raised in any of our subjects, supporting the findings of Siegel et al, who used the same method of analysis. ${ }^{12}$ This may reflect the increased specificity of this third generation assay for troponin I, as previous studies could have had positive results for troponins owing to cross reaction with skeletal muscle troponins. The participants used in this study came from a diverse range of ages, athletic abilities, and training states, in contrast to some other studies that looked at elite athletes. The type of exercise may also be important, as endurance cycling or multidisciplinary events such as triathlon may put different stresses on the myocardium to pure endurance running events, and comparisons between them may therefore be inappropriate. The debate as to whether exercise causes leakage of myocardial cell contents,

Table 2 Summary of the results before and after exercise, giving normal ranges

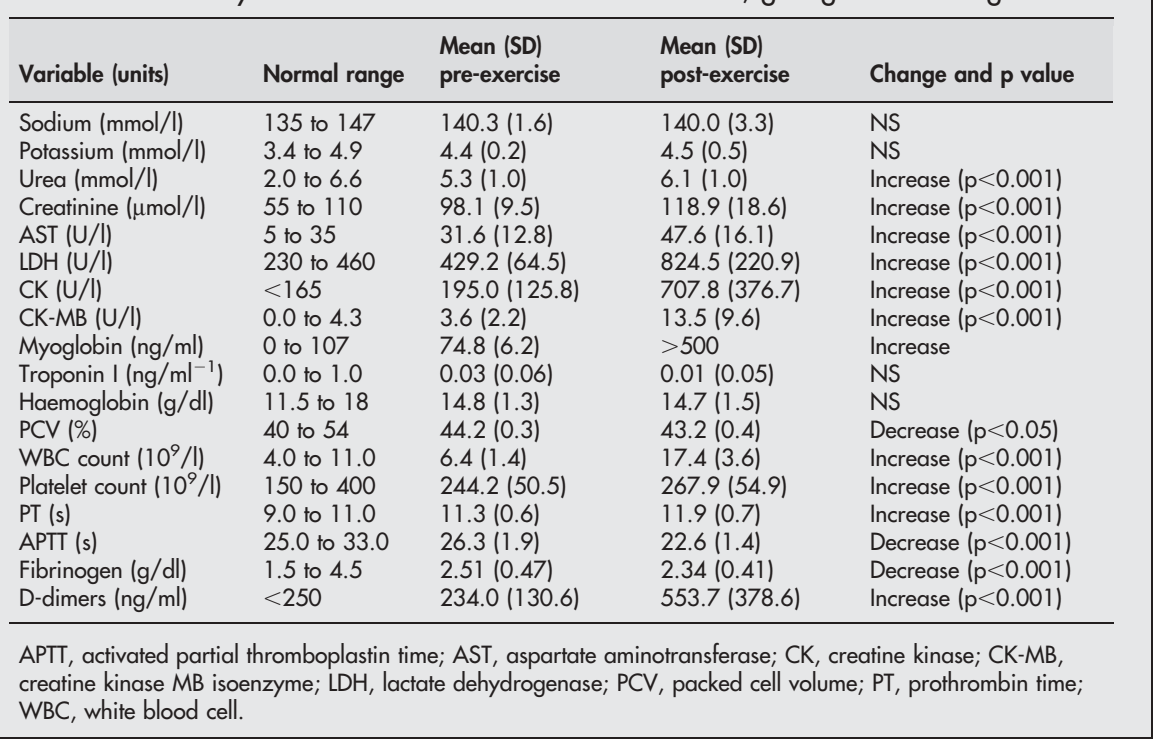




\section{Take home message}

Many of the diagnostic biochemical and haematological tests on which we rely are affected by exercise, and physicians should be aware of these effects when interpreting results in the clinical setting. Muscle enzymes, white blood cell count, and markers of clotting and fibrinolysis in particular may be abnormal as a result of exercise.

and whether there is true myocardial cell damage or temporary fatigue of the myocardium is ongoing.

The relation between training status and resting CK has already been described, ${ }^{5}$ and this study confirms that there is a positive correlation between training status and resting CK and CK-MB level. It also shows that the percentage change in $\mathrm{CK}$ and $\mathrm{CK}-\mathrm{MB}$ following exercise is higher in the less trained athletes, despite their running slower race times, confirming observations from previous work. ${ }^{13}$

Haematological indices were shown to change following exercise, and may cause some confusion in the interpretation of the results in a hospital setting. A markedly raised white cell count $\left(6.4(1.4) \times 10^{9} / 1\right.$ pre- to 17.4 (3.6) post-exercise, $\mathrm{p}<0.001$ ) was caused predominantly by a neutrophilia, and may be confused with an infective or inflammatory process. Similar exercise induced changes have been documented in previous studies. ${ }^{14}$

Haemostasis is also affected by exercise, with activation of both clotting and fibrinolysis. With particular relevance to the laboratory investigations identified, products of fibrinolysis such as D-dimers have been shown to rise as a result of marathon running, ${ }^{15}{ }^{16}$ and this was confirmed by our findings. This may be mistakenly taken to signify a thromboembolic disorder in a clinical setting. It has been suggested that unequal activation of the clotting and fibrinolytic cascades may result in a tendency to form intravascular clot, although other effects of exercise, namely vasodilatation and increased blood flow, should counteract this in normal healthy individuals. The temporal relation between the relative activation of these cascades may yet yield a clue in the search for the cause of some cases of exercise related sudden cardiac death, and occasional exercise related thrombotic cerebrovascular events in runners found to have normal cerebral arteries.

\section{Conclusions}

Owing to the potential effects of exercise on biochemical and haematological variables, we suggest that a full history is essential before interpreting any abnormality in these indices, in order to avoid the marathon runner being mistakenly confined to a hospital bed on spurious grounds.

\section{ACKNOWLEDGEMENTS}

The study was supported by the London Marathon. We would like to thank Dr Roger Amos of Homerton Hospital for his help in the processing of samples, and Mr David Munyard of Axis Shield Diagnostics Limited for his cooperation and loan of equipment.

\section{Authors' affiliations}

J E Smith, Academic Department of Sports Medicine, Royal London Hospital, London EI, UK

G Garbutt, Philippe Lopes, School of Health Sciences, University of East London, Dagenham, Essex, UK

Dan Tunstall Pedoe, Department of Cardiology, Homerton Hospital, London E9, UK

\section{REFERENCES}

1 Herren KR, Mackway-Jones K. Emergency management of chest pain - a review. Emerg Med J 2001;18:6-10.

2 Becker DM, Philbrick JT, Bachhuber TL, et al. D-dimer testing and acute venous thromboembolism. A shortcut to accurate diagnosis? Arch Intern Med 1996;156:939-46.

3 Bernardi E, Prandoni P, Lensing AW, et al. D-dimer testing as an adjunct to ultrasonography in patients with clinically suspected deep vein thrombosis: prospective cohort study. BMJ 1998;317:1037-40.

4 El-Sayed MS, Sale C, Jones PGW, et al. Blood hemostasis in exercise and training. Med Sci Sports Exerc 2000;32:918-25.

5 Priest JB, Oei TO, Moorehead WR. Exercise-induced changes in common laboratory tests. Am J Clin Pathol 1982;77:285-9.

6 Kargotich S, Goodman C, Keast D, et al. The influence of exercise-induced plasma volume changes on the interpretation of biochemical parameters used for monitoring exercise, training and sport. Sports Med 1998;26:101-17.

7 Van Beaumont W, Underkofler S, van Beaumont S. Erythrocyte volume, plasma volume and acid-base changes in exercise and heat dehydration. $J$ Appl Physiol 1981;50:1255-62.

8 Siegel AJ, Silverman LM, Holman BL. Elevated creatine kinase MB isoenzyme levels in marathon runners. JAMA 1981;246:2049-51.

9 Rogers MA, Stull GA, Apple FS. Creatine kinase isoenzyme activities in men and women following a marathon race. Med Sci Sports Exerc 1985; 17:679-82.

10 Whyte GP, George K, Sharma S, et al. Cardiac fatigue following prolonged endurance exercise of differing distances. Med Sci Sports Exerc 2000;32:1067-72.

11 Rifai N, Douglas PS, O'Toole M, et al. Cardiac troponin T and I, echocardiographic [correction of electrocardiographic] wall motion analyses, and ejection fractions in athletes participating in the Hawaii Ironman Triathlon. Am J Cardiol 1999;83:1085-9.

12 Siegel AJ, Lewandrowski EL, Chun KY, et al. Changes in cardiac markers including B-type natriuretic peptide in runners after the Boston Marathon. Am J Cardiol 2001;88:918-20.

13 Noakes TD, Carter JW. The responses of plasma biochemical parameters to a $56-\mathrm{km}$ race in novice and experienced ultra-marathon runners. Eur J Appl Physiol 1982;49:179-86.

14 McCarthy DA, Dale MM. The leucocytosis of exercise: a review and model. Sports Med 1988;6:333-63.

15 Rocker L, Taenzer M, Drygas WK, et al. Effect of prolonged physical exercise on the fibrinolytic system. Eur J Appl Physiol 1990;60:478-81.

16 Prisco D, Paniccia R, Bandinelli $B$, et al. Evaluation of clotting and fibrinolytic activation after protracted physical exercise. Thromb Res 1998;89:73-8. 